\title{
Reflexión sobre enfoques y métodos utilizados en la ciencia de los transportes
}

\section{Guidelines on the approaches and methods used in transportation science}

\author{
Óscar SÁNCHEZ-Flores* \\ ANDRÉ De PALMA**
}

\begin{abstract}
This paper provides a critical analysis about the currently methodologies and modeling approaches used in transportation science. The inconsistency between practice, modeling techniques and recent theoretical development in other disciplines, are pointed out in this contribution. Four key axes of analysis are proposed: modeling individual behavior from social psychology and micro-psychology approach, modeling individual behavior from neoclassical microeconomic approach, the limitations of deterministic formulation in transportation problems vis-à-vis from stochastic formulation for which we bet on and the static and dynamic formulation, in order to show that last one is more suitable for transportation problems analysis.
\end{abstract}

Keywords: methods, modeling, transportation systems.

\section{Resumen}

Se presenta un análisis crítico sobre las metodologías y enfoques de modelación utilizados actualmente en la ciencia de los transportes. Se señalan inconsistencias que surgen de la confrontación entre la práctica, las herramientas de modelaje utilizadas y los desarrollos teóricos en varias disciplinas. Se plantean cuatro ejes de análisis: la modelación del comportamiento del individuo según la perspectiva de sicología social y en micro-sicología; el enfoque clásico de análisis del comportamiento propuesto en economía; la formulación determinista vis-à-vis en contraste con la formulación estocástica, tomando partido por esta última, y la formulación estática versus la dinámica, optando por esta última.

Palabras clave: métodos, modelación, sistemas de transporte.

* Benemérita Universidad Autónoma de Puebla. Correo-e: oscar.sanchezflores@correo.buap.mx

** L'Ecole Polytechnique y Ecole Normale Supérieure de Cachan, Francia. Correo-e: andre. depalma@ens-cachan.fr 


\section{Introducción}

El análisis de los fenómenos del transporte puede ser definido como una disciplina científica que trasciende la tecnología y los métodos, ya que se requiere entender sus propiedades fundamentales. En este sentido, la ciencia de los transportes permite identificar estas propiedades demostrando cómo el conocimiento de un elemento del sistema de transporte puede utilizarse para entender el comportamiento de otro elemento o subsistema.

En esta ciencia, como en otras de las ciencias naturales, existe también la necesidad de entender los fenómenos cotidianos que suceden a nuestro alrededor (Hall, 2003). Por ejemplo, en la década de los sesenta este interés se orientaba a la dinámica del flujo vehicular en las carreteras o ciudades. Actualmente, este fenómeno no sólo se analiza como el resultado del desplazamiento de una persona o un grupo de mercancías, sino que también se busca entender la interacción con la infraestructura, la interacción de las variables propias del flujo vehicular, la formación y disipación de filas de espera, así como de los cuellos de botella, y finalmente, la diversidad de comportamientos de los agentes (usuarios, conductores, no usuarios, reguladores, empresas) que inciden no sólo en el funcionamiento del sistema de transporte, sino que también interactúan con él en diferentes horizontes temporales.

De esta manera, la motivación de los científicos actuales del transporte reside en entender los fenómenos asociados a las interacciones espaciales, temporales, económicas y sociales que surgen del traslado de personas, bienes o cosas de un lugar a otro; para ello, emplean tradicionalmente el conocimiento desarrollado previamente en disciplinas como la geografía, economía, teoría de la localización, así como metodologías propias de la física, investigación de operaciones, teoría de probabilidades y del control. Posteriormente extienden, desarrollan y aplican estos conocimientos en el análisis de los fenómenos de transporte; se trata, por lo tanto, de una disciplina cuantitativa que se apoya, en general, en modelos matemáticos y algoritmos de optimización para entender y explicar los fenómenos propios del transporte (Hall, 2003). Las aportaciones al conocimiento se logran a partir de análisis teóricos y empíricos (o ambos) en laboratorios reales que son los sistemas de transporte en operación, o en su caso, hacen abstracciones de todo el entorno en el que operan (modelación), para entender y prever comportamientos futuros de los agentes referidos que pueden ser utilizados para la toma de decisiones que lleven a mejorar la eficiencia de estos sistemas o sus indicadores de desempeño. En este sentido, el objetivo principal de este artículo es aportar un análisis crítico sobre las metodologías y enfoques de modelación utilizados actualmente en la ciencia de los transportes. Se explora, en específico, la trasposición 
de conocimientos de las ciencias físicas, la fisicoquímica, la economía y las matemáticas aplicadas al análisis de los fenómenos propios de los sistemas de transporte, enfatizando en los tópicos que no han sido plenamente tratados y, en su caso, abordando el sentido y legitimidad de este procedimiento.

De este análisis se pretende motivar la reflexión relacionada con las limitantes y alternativas para subsanar los métodos y enfoques de modelación utilizados en transportes. Dicha reflexión se inscribe en el marco de trabajos anteriores descritos en De Palma y Pahaut (1989 y 1999); De Palma (1998) y Ortúzar y Sánchez (2004).

Este artículo pudiera ser considerado de alguna manera dogmático. La econometría, la investigación de operaciones, las ciencias económicas y los transportes no siempre se asocian bien. Los autores consideran que el objetivo de la modelación no sólo es desarrollar abstracciones que permitan entender o replicar un fenómeno que, a su vez, pudiera ser una herramienta útil en la práctica, sino también se requiere que éstas sean consistentes en su formulación (consistencia interna) y en la caracterización del problema (consistencia externa); es decir, herramientas cuyas bases, teóricas o empíricas, sean sólidas; este rigor induce un costo, efectivamente, pero permite también dominar los desbordamientos descontrolados de la actividad de modelación, entendiendo esta última como el proceso secuencial que inicia con la construcción de un modelo semántico que sólo incluye conceptos y relaciones para convertirse en un modelo formal cuando se incluyen variables explicativas del fenómeno caracterizado, de ahí a transformarse en un modelo resoluble cuando se agrega una fórmula característica que implica una técnica de estimación y, finalmente, un modelo alimentado, cuando una vez superados los niveles anteriores, se introducen datos correspondientes a una realidad observada, y se ajusta para replicar un sistema determinado (Ortúzar y Sánchez, 2004).

En efecto, el poder de cálculo, cada vez más considerable a nuestra disposición, nos conduce con mayor frecuencia a olvidar el principio de precaución en el proceso de modelación y a explicar de manera insuficiente las suposiciones muy a menudo implícitas en los modelos propuestos. Este último tipo de enfoque pragmático, aceptado en un contexto no académico, no parece justificado en una perspectiva de investigación de largo plazo y que aspira a ser acumulativa. Más que proponer respuestas o incluso procedimientos a seguir, los autores prefieren resaltar ciertas inconsistencias que surgen de la confrontación en la práctica en materia de transportes, las herramientas de modelaje utilizadas para responder a los cuestionamientos inducidos por este contexto práctico y el arsenal teórico a nuestra disposición. 
En la sección siguiente se aborda la modelación del comportamiento tal como ha sido inicialmente desarrollado en sicología social y en microsicología. En la tercera parte se analiza el enfoque clásico del análisis del comportamiento propuesto en economía, así también se muestran las fortalezas y debilidades de este enfoque en el contexto de las ciencias de los transportes. En el cuarto apartado, más técnico, se aborda la cuestión de la formulación determinista vis-à-vis de la formulación estocástica por la que se toma partido. La sección quinta compara la formulación estática y dinámica mostrando argumentos de la pertinencia de ésta última. Las conclusiones que resultan de los cuatro ejes de análisis son reagrupadas en la última sección de este artículo.

\section{La teoría del comportamiento}

Comenzaremos por describir un modelo básico de comportamiento individual: todo sistema de transporte es el rencuentro entre la suma de comportamientos individuales (demanda) y las tecnologías existentes (oferta), en general, se asume que todo individuo actúa en beneficio de sus intereses, aunque esta gran verdad pierde sentido para el matemático, en efecto, decir que un individuo optimiza algo pudiera parecer puramente tautológico, en tanto no se especifique lo que se optimiza; sin embargo, esta afirmación posee un contenido confuso, dado que contiene una serie de suposiciones implícitas, por ejemplo, supone que el individuo puede ser visto como un operador capaz de calcular y que las decisiones que toma son consecuencia de este cálculo, dicho supuesto tiene su origen en la teoría utilitarista que inspiró después a los estudiosos de la teoría económica, en la cual, el individuo es impulsado a decidir y tales determinaciones pueden ser clasificadas aun como cotidianas y de baja recurrencia, estructuradas a partir un árbol de decisión cronológica que se extiende del muy corto (elección del modo de transporte, de la hora de inicio de un viaje, etc.) al largo plazo (decisiones de localización del hogar) en donde estas últimas condiciones influyen en las primeras.

Nótese que a menudo las decisiones presentan al individuo de manera exógena, ya que, en efecto, tomar una decisión bajo este enfoque obliga a excluir alguna de las alternativas existentes, lo que corresponde a renunciar a algo cuyo costo o beneficio no necesariamente se puede evaluar, lo cual implica que la decisión no será tomada de manera racional, a no ser que el costo de no decidirse sea superior al costo de tomar la decisión; además, es necesario estimar correctamente los costos; esta evaluación es por sí misma onerosa ya que se requiere de un esfuerzo cognitivo y de memoria, es decir, este cálculo implica un conjunto de renuncias o exclu- 
siones que el individuo debe aceptar con mayor o menor intensidad y de manera más o menos amplia.

La ciencia no parece todavía aportar respuestas satisfactorias a esta serie de cuestionamientos sin fin: ¡es necesario calcular?, ¿cuánto tiempo es necesario calcular?, etc.; estas preguntas resultan de por sí difíciles de responder, y más aún, tratándose de un individuo aislado. Una vez que los individuos entran en interacción, de manera tal que el beneficio de uno depende de las acciones de otros, la complejidad de este tipo de problemas se bifurca. La teoría de juegos se ha dedicado a aportar respuestas a alguna de las cuestiones planteadas; sin embargo, son arduas y poco prácticas para modelar problemas de gran tamaño, como es el caso de los sistemas de transporte, en donde intervienen agentes con múltiples intereses.

La ciencia de los transportes también ha abordado estos problemas guiada más por la voluntad de desarrollar herramientas operacionales que puedan ser puestas en marcha y probadas empíricamente que por modelos formales y consistentes. Los diferentes enfoques o corrientes de pensamiento que tratan de aportar respuestas en este sentido se ignoran profundamente, salvo algunas excepciones como son las que se mencionan a continuación.

En la teoría del comportamiento referida, el ente o agente decisor, desde una visión simplificadora, pero rica en consecuencias, tiende a reducir en la medida de las posibilidades los costos asociados a sus actos, optimizando de la mejor manera sus beneficios. En micro-sicología se distinguen cuatro tipos de costos: monetarios, energéticos, temporales y sicológicos (Moreno, 1970; Moles y Rohmer, 1977). En el marco de elección modal entre el automóvil y el transporte público, por ejemplo, el costo monetario de emplear el primer modo, corresponde principalmente al precio del carburante y al costo del uso del automóvil; en tanto que para el segundo, es el boleto. El costo energético corresponde al trayecto a pie o al desplazamiento vertical para cambiar de nivel de circulación, en tanto que el costo temporal para el automóvil está relacionado con el tiempo consumido en el vehículo (automóvil o autobús), la espera, el tiempo de acceso al vehículo o al destino. Finalmente, el costo sicológico es el relativo a la incertidumbre del tiempo de viaje ocasionada por la congestión o por la hora de llegada de un transporte público, es decir, elementos asociados a la presencia de cierto riesgo.

En lo que respecta a la evaluación de los beneficios, el problema es aún más delicado: si los costos parecieran ser juzgados al mismo equivalente monetario, no sucede lo mismo con los beneficios. Esta asimetría en la facilidad de cuantificación se refleja en la práctica de la modelación: para un modelo de elección modal, por ejemplo, los costos son habitualmen- 
te descritos de manera muy detallada, mientras que los beneficios se expresan con la ayuda de un parámetro simple que debiera capturar por sí solo la diversidad de cualidades otorgadas para una alternativa de elección.

Pareciera entonces que la investigación debe dar algunos saltos hacia adelante. En efecto, es probable que la serie de beneficios a los que el individuo aspira (por ejemplo, al momento de realizar un desplazamiento) sea susceptible de descomponerse por una serie de partes elementales que respondan al esquema clásico en marketing necesidad-deseo-demanda, éstas, una vez identificadas, deberían poder combinarse por una álgebra que les sea propicia. En el mismo sentido, trabajos recientes sobre el tema de la elección modal se encaminan hacia la medición de la calidad del servicio como beneficio. Siendo esta variable intangible de valoración subjetiva, la funcionalidad de este enfoque lleva a la necesidad de combinar el conocimiento de otras disciplinas (Lecourt, 2010), como es el caso de la teoría de elecciones ordinales (Hensher y Grenne, 2010) en la que se asocian las teorías del comportamiento del consumidor con aquellas del marketing.

\section{La teoría neoclásica del comportamiento y sus límites}

La economía ha propuesto un conjunto de axiomas estricto para describir el comportamiento de los individuos. En esta sección se examinan sus virtudes y sus vicios.

Las preferencias de los individuos pueden, en economía, traducirse bajo forma de relaciones de preferencias que satisfacen axiomas -reflexibilidad, transitividad, etc- (Ben-Akiva y Lerman, 1985). Se ha mostrado que en esos casos, las preferencias pueden expresarse en forma de funciones de utilidad cuasi-cóncavas que traducen de manera numérica (relación cardinal) las relaciones de preferencia (ordinal) de los individuos; someramente, en la práctica se expresa esta función de utilidad como una función de satisfacción en forma de una diferencia entre los costos y beneficios asociados a cada opción.

El origen de estas funciones de utilidad es poco claro, éstas aparecen frecuentemente especificadas en formas cómodas desde el punto de vista matemático para su resolución, sin relación directa entre ésta y la sicología, o aún menos, con el inconsciente del individuo. De hecho, el argumento "todo sucede como si..." se vuelve cada vez menos satisfactorio y justificado. La tendencia, tanto en economía industrial como en economía de transportes, es intentar comprender el fundamento de estas funciones de utilidad, es decir, de abrir esa caja negra. Como prueba de ello se citan las recientes incursiones en economía experimental. Paralelamente, los axiomas de base de la teoría de la elección empiezan a cuestionarse; por ejemplo, 
el axioma de transitividad es violado en el enfoque de los modelos de elección discreta que son utilizados (y validados) de manera rutinaria en el dominio de los transportes. En este sentido, se proponen tres diferentes tipos de apertura de la caja negra relacionados a la metodología específica de la economía neoclásica. Los tres parecen esenciales para el modelador en el campo de los transportes, ya que se desea sostener la idea que el enfoque económico en materia de modelación de la demanda de transportes es adecuado a condición que éste sea debidamente corregido.

\subsection{Primera apertura: hacia la desagregación}

\subsubsection{El individuo representativo y el conjunto de individuos}

Una primera apertura se ha realizado en el momento que el paradigma del individuo representativo (el individuo medio de Quételec) ${ }^{1}$ ha sido puesto en tela de juicio, tanto desde el punto de vista teórico como empírico; en efecto, ¿̨uál es el proceso de agregación de preferencias subyacente a la definición de un individuo representativo? Como la intuición lo sugiere, una cantidad importante de información se pierde cuando los datos individuales se agregan (Boursin, 1995). Aún más, las nuevas tecnologías tienden a poner a disposición datos cada vez más desagregados, como es el caso del uso de teléfonos celulares, que registran la información de la ubicación espacial de las personas, de los cookies que permiten seguir la huella de los comportamientos de exploración de los visitantes de un sitio de internet, o bien, las tarjetas de prepago que permiten conocer aspectos específicos de la movilidad de las personas en transporte público.

Por otro lado, la potencia de cálculo y de memoria, cada vez más importantes de las herramientas computacionales, permite actualmente tratar datos desagregados de varios millones de individuos. En el caso de los sistemas de transporte, actualmente, esta disponibilidad de información desagregada por individuo ha inducido la aparición de enfoques de análisis emergentes que tratan de entender y prever el comportamiento de las personas, pasando de un análisis tradicional de viaje aislado caracterizado por un par origen-destino (Ortúzar y Willumsen, 2001), a uno de actividades que considera que el conjunto de viajes de una persona se derivan de la organización de estas actividades, las cuales se encuentran limitadas por las horas de un día (Etemma y Timmermans, 1987, Ben-

\footnotetext{
${ }^{1}$ Es importante mencionar la existencia de otros intentos de modelación matemática en el que se establece que los números pequeńos y los problemas de agregación de preferencias tuvieron lugar alrededor del periodo revolucionario 1720-1820. En este caso, fueron los problemas propuestos (principalmente por los juegos de azar, por los tribunales con los sistemas de prueba y semiprueba) que provocaron la producción de herramientas matemáticas. Curiosamente, este enfoque fue abandonado por el de grandes números y la investigación de las regularidades estadísticas.
} 
Akiva y Bowman, 2001). Las fuentes de información para este tipo de análisis pueden provenir de bases de datos obtenidas con las nuevas tecnologías (Stopher y Stecher, 2006; Wachowicz, 2010) y más recientemente del análisis de redes sociales (Wasserman y Faust, 1999; Carrasco y Miller, 2009).

Una primera línea de trabajo consiste en enjuiciar la descripción abstracta del individuo representativo o medio, para concentrarse en una descripción individual del comportamiento. Este enfoque desagregado parte de una perspectiva más intuitiva, aportando beneficios desde un punto de vista empírico; sin embargo, posee en contraparte los inconvenientes siguientes: ¿cómo describir teniendo en cuenta la heterogeneidad de comportamientos? ¿cómo pasar de la descripción detallada de un individuo a una descripción agregada de la población (el problema tipo de agregación)? La primera pregunta plantea cómo diferenciar a los individuos de forma operacional (no sólo según su categoría socioeconómica, sino también el nivel cognitivo, experiencia pasada, carácter, etc.). En el enfoque agregado, difícilmente se toman en cuenta estas fuentes de diferenciación de las personas. La segunda línea de trabajo por desarrollar corresponde a la agregación y es mucho más difícil que la primera, dado que el comportamiento de los usuarios no puede adicionarse simplemente. Los usuarios de los servicios sujetos a congestión, como son los sistemas de transporte, están evidentemente en interacción. El tiempo de recorrido sobre un itinerario urbano determinado, por ejemplo, es resultado de las decisiones de elección de itinerarios del conjunto de los usuarios, de las interacciones que se dan entre los vehículos y la infraestructura (características geométricas y capacidades). Aunque estas dos fuentes de dificultad sean importantes, parecen más fácilmente abordables que aquellas que surgen cuando el modelador tiene como objetivo describir simplemente las leyes que rigen el comportamiento de un grupo de individuos (enfoque del consumidor o del usuario representativo).

\subsubsection{Efectos perversos}

Cuando se tiene un conjunto de fenómenos asociados que pretende comprenderse se requiere una descripción suficientemente detallada de cada uno de ellos. A partir de esta consideración, es de llamar la atención que la noción de red haya interesado tan poco a los economistas, a excepción de la época de M. Beckmann et al. (1956) y P. Samuelson (1952). Los economistas tratan bien el tema de externalidades de red; sin embargo, proponen para hacerlo una representación en la que la idea de red está sorprendentemente ausente. Si se toma un caso simple para el cual la representación desagregada de la oferta es fundamental, esta desagregación 
pasa por la idea de red. Si el número de usuarios que utilizan una infraestructura es definido, se podría afirmar que un aumento (supuestamente gratuito) de la capacidad de la infraestructura no debería aumentar los costos que los individuos soportan. Por muy intuitiva que parezca esta afirmación (aunque no sea opuesta a las leyes fundamentales de la economía neoclásica), no es menos falsa, como lo muestra la famosa paradoja de Braess (1968) y (Braess et al., 2005).

Para demostrarlo, se aporta una versión simplificada de esta paradoja que tiene la ventaja de ser más simple: tomemos el caso de las poblaciones $A$ y $B$ unidas a la ciudad $V$ por medio de dos caminos (ver figura I): $A V$ (de $A$ a $V$, de gran capacidad y con un tiempo de trayecto de una hora) y $B V$ (de $B$ a $V$ de capacidad reducida pero suficiente para que los habitantes de $B$ puedan viajar a la velocidad máxima permitida con un tiempo de trayecto estimado de 30 minutos). Como los caminos constituyen el único transporte para acceder a la ciudad, el tiempo medio de recorrido será una media ponderada (por el número de personas de cada población) del tiempo de trayecto de cada uno de los caminos $A V$ y $B V$. Supongamos ahora que se construye un camino mucho más rápido de la población, de $A$ a $B$, de cinco minutos. Los habitantes de $B$ tienen la posibilidad de tomar la ruta $B V$ o los tramos $B A$ y $A V$ pero no les interesa porque ocuparían 65 minutos para llegar a $V$. Por el contrario, una parte de los habitantes de $A$ van a utilizar el nuevo tramo $A B$ y el camino $B V$ ya que ello les redituaría en un tiempo menor al actual, de una hora. Si las dos alternativas posibles para llegar a $V$ son utilizados por los habitantes de $A$, resulta que los tiempos de trayecto de $A V$ y de los tramos $A B$ y $B V$ son necesariamente los mismos, de suerte que el nuevo tiempo de trayecto de $B V$ (estado de equilibrio) será de 55 minutos. Esto se debe al hecho que los habitantes de $A$ han elegido la ruta $B V$ hasta que el tiempo de trayecto sobre los tramos $A B$ y $B V$ sea el mismo que el del tramo $A V$. Como la capacidad de $A V$ es grande, el tiempo de trayecto en ésta permanece constante y los habitantes de $A$ no se benefician, inicialmente, de la ruta más rápida $B V$ puesto que está saturada, de hecho, el tramo nuevo no les hace ganar ni perder tiempo; por el contrario, para los habitantes de $B$ el tiempo de trayecto aumenta de tal manera que el beneficio para el conjunto de usuarios disminuye.

Esta constituye la versión más simple de la paradoja de Braess, muestra que el tiempo de equilibrio de un sistema de transportes puede aumentar cuando una nueva ruta se construye dentro de un sistema. La razón teórica de este fenómeno resulta del hecho de que los individuos siguen una regla de comportamiento individual y egoísta de optimización del interés propio, sin preocuparse del beneficio social. Los habitantes de $A$ utilizan la ruta $B V$ sin preocuparse que ello demore a los habitantes de 
$B$. La presencia de externalidades, es decir, de costos sociales que no son tenidos en cuenta explícitamente por los usuarios, explica la existencia de efectos perversos de este tipo. De manera similar, una empresa que contamina puede inducir a disfuncionamientos similares en la sociedad. Dicho en otros términos, el excedente social del consumidor puede aumentar cuando se le aplica un impuesto a un bien rentable para una empresa; éste será cierto, siempre y cuando, el producto en cuestión sea creado en asociación con otros elementos contaminantes que contribuyen a aumentar el costo social, por ejemplo, aumentando los costos de producción de otras empresas.

\section{Figura I}

\section{Ilustración de la paradoja de Braess}

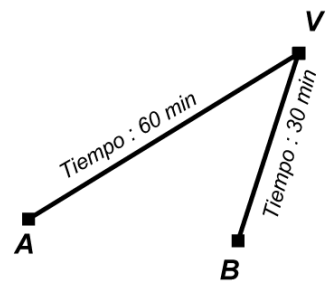

Dos carreteras van a $V$



Se construye tramo nuevo $(A-B)$

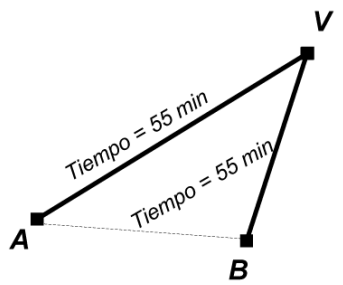

El tiempo de BV aumenta

Fuente: Elaboración propia.

\subsection{Segunda apertura: preferencias endógenas}

Una segunda apertura opera a partir del momento que el modelador incluye en el problema las preferencias endógenas, esta cuestión no ha sido abordada directamente por los especialistas del transporte, por lo que constituye un tema de investigación innovador; en efecto, es razonable decir que los hábitos de movilidad no son el resultado de preferencias puramente exógenas como se considera en la práctica actual de la modelación. Las instituciones, normas, culturas y religiones, así como la memoria colectiva, vinculadas a la estructura urbana, inducen relaciones de preferencias y poseen probablemente una solidez superior y una capacidad de resistencia a la erosión del tiempo más pronunciada. A este respecto, el enfoque en términos de preferencias endógenas está llamado a desempeñar un papel más importante en la modelación del comportamiento de los usuarios en razón de su carácter invariable; sin embargo, estas ideas son, por ahora, especulativas en el entorno de los transportes, puesto que no ha sido probado ni teórica ni empíricamente, quedando abiertas para 
investigaciones futuras que se ofrecen sin demora a las nuevas generaciones de investigadores.

\subsection{Tercera apertura: interacción}

La tercera apertura está ligada al estudio de la interacción entre los individuos y ha sido explorada de diferentes maneras, que pueden agruparse en alguno de los dos niveles siguientes:

\subsubsection{Primer nivel}

Por un lado, los individuos pueden responder a la misma variable de costo que es el resultado de agregar sus decisiones individuales. En este sentido, se cita la definición de congestión de Mohring (1999:182): "Congestion can be defined as occurring when customer-supplied inputs per unit if output depend on the rate at which a fixed facility is utilized". En este caso, la interacción de los vehículos con la infraestructura se explica a través de variables macroscópicas de tráfico que por ellas mismas son funciones de agregación de decisiones individuales (el flujo vehicular y la velocidad media).

Desde este punto de vista, la cinética social se opone a la cinética química puesto que en este último caso las interacciones son esencialmente locales, es decir, de corto alcance espacial. En el caso de los transportes, es cierto que la decisión del automovilista sobre una ruta depende a menudo de interacciones locales como lo han tratado de reflejar la teoría del automóvil líder-seguidor y los estudios de la congestión basados en los autómatas celulares (Gazis, 2002), trasmisión por celdas (Daganzo, 1994), entre otros. Sin embargo, se trata de una diferencia entre un sistema químico y un sistema social que puede ilustrarse cuando un automovilista decide desviarse y utilizar un itinerario alternativo, una vez que recibe el mensaje de la radio sobre las condiciones de circulación, respondiendo con ello a una lógica de interacción no local.

\subsubsection{Segundo nivel}

El otro nivel proviene de considerar que los individuos que están en interrelación también tienden a cambiar su experiencia y preferencia. Este tema, que implica también la determinación de preferencias endógenas, no ha sido, curiosamente, objeto de investigaciones en el campo de los transportes. Por consiguiente, las preferencias sobre la utilización del automóvil para desplazarse al trabajo o para hacer las compras, no son nada más que decisiones parcialmente individuales. Éstas implican tam- 
bién un efecto de moda, la cual induce una serie de ordenamientos de la infraestructura impulsada al mismo tiempo por las modas. La predicción a largo plazo pasa, al parecer, por la explicación de estos mecanismos robustos y por el estudio de sus implicaciones.

\subsubsection{Externalidades de red}

Los procesos de interacción, descritos parcialmente por los economistas (que han olvidado a menudo la componente tecnológica de las interacciones), se han concentrado en las implicaciones de estos procesos en términos de la teoría de los juegos. Hablamos de externalidades positivas (o negativas) de red cuando el beneficio ligado a la utilización de un bien aumenta (o disminuye) con el número de usuarios de este bien. Esta relación de interdependencia puede ser directa, como en el caso del congestionamiento de un camino, o indirecta, como en el caso de las tarjetas de crédito.

Describamos primeramente la externalidad directa: cuando dos caminos unen un mismo punto de origen y destino, al equilibrio, el número de usuarios para cada una de las rutas está determinado por la igualdad de los tiempos de trayecto de cada una de ellas. El tiempo de trayecto de cada camino depende del número de usuarios. Esta externalidad puede traducirse también de manera indirecta: la utilidad de una tarjeta de crédito aumenta con el número de usuarios, esto se debe a que cuando la cantidad de usuarios es mayor, el número de comerciantes susceptibles de aceptar dicha tarjeta se incrementa; por consiguiente, el potencial de utilización de la tarjeta aumenta indirectamente.

En la discusión anterior se ha considerado también un tercer tipo de relación que proviene de fenómenos de influencia, imitación, del seguimiento de una norma, etc. Todo proceso de interacción puede explicar el proceso de elección individual. Subrayamos que pocos trabajos han sido desarrollados en este sentido; sin embargo, este tipo de investigación es susceptible de aportar los elementos de reflexión y posteriormente de modelaje para disponer una descripción robusta del largo plazo, investigaciones cuyos resultados son todavía deficitarios en materia de transportes.

\section{A propósito de la escala}

En esta sección se desarrolla una reflexión relacionada con la definición de sistema, con la posibilidad de tratar una parte representativa de él y con la de borrar los elementos aleatorios que afectan necesariamente todo proceso de modelación, (Schelling, 1980). 


\subsection{Interacciones locales y no locales}

Considere el ejemplo que muestra la peligrosidad de hacer uso de la ley de los grandes números para eliminar las fluctuaciones que afectan necesariamente el comportamiento de los modelos.

Pensemos en un sistema químico: los procesos de interacción en química son típicamente locales, esto quiere decir a la escala molecular. Las leyes de interacción molecular son fuertes pero de corto alcance, contrariamente a las leyes de interacción gravitacionales que son comparativamente de una intensidad extraordinariamente baja. ${ }^{2}$ Esta condición de efecto local de las interacciones desempeña un papel esencial en la derivación de leyes de la cinética química, lo que permite describir el comportamiento de una molécula como el resultado de la acción de las moléculas vecinas únicamente.

Esta constatación corresponde, en efecto, a una simplificación de la descripción. En contraparte, induce al cuestionamiento del desarrollo de la estructura organizada a la escala del ser humano (en la cual el metro sería, por ejemplo, la magnitud característica), escala gigantesca en comparación con la talla de las moléculas. Esta cuestión ha sido abordada en el marco de las estructuras disipativas, o por el método de análisis de los campos (Modelo de Ising). Sin embargo, en el caso de las ciencias sociales, se ha buscado realizar transposiciones mecánicas de la física o de la físico-química. Por fortuna existen contra ejemplos notables, en particular en el área de los transportes. El paso de la escala microscópica (nivel individual) al nivel macroscópico (nivel agregado) ha sido ilustrado por los estudios de la congestión basados en el modelo del automóvil líderseguidor. En efecto, se supone que cada vehículo regula su velocidad en función de los vehículos que lo rodean. Resultando que las interacciones son locales. Las ideas básicas de descripción de la congestión con ayuda de autómatas celulares estocásticos consideran los mismos conceptos de corto alcance geográfico. En el caso del estudio de la congestión, el resultado aportado por el modelo es la derivación de leyes agregadas entre un número de vehículos y la velocidad media de circulación. El cambio de escalas es mucho menos espectacular que para el caso de la físico-química, en el que se pasa de una molécula (nivel individual) a $10^{23}$ moléculas (en el caso macroscópico) mientras que en el tráfico se pasa modestamente de algunos vehículos a una centena o millares de unidades.

Recientemente se ha realizado un análisis que consiste en estudiar cómo las leyes que describen el nivel de equilibrio de la congestión pu-

\footnotetext{
${ }^{2}$ En efecto, se ha llegado a reproducir las acciones entre las moléculas que se oponen a la gravitación universal y que en el presente caso emana de un cuerpo extremadamente voluminoso: la Tierra.
} 
dieran ser transpuestas a la escala de una ruta y al nivel de una población. Más específicamente, se ha mostrado que los resultados obtenidos en el marco del Modelo de Vickrey (1969) para un origen, un destino y una ruta, podían ser ampliados numéricamente para el caso de una población caracterizada por una matriz origen-destino incluyendo varias centenas de entradas y una red de transportes compuesta de varios millares de ligas o arcos (De Palma y Marchal, 2002).

Los dos casos citados, uno relativo a la agregación con la aparición de leyes a nivel macroscópico que no tienen equivalencia a nivel microscópico, y el otro, correspondiente al caso de la transposición de una ley de una escala a otra, presentan el mismo cuestionamiento desde el momento que nos ocupamos del carácter operacional de estas leyes agregadas. Así, los parámetros obtenidos a nivel macroscópico tienen idealmente que estar ligados al nivel de detalle microscópico, puesto que estos parámetros tienen una interpretación simple e intuitiva. Por ejemplo, la capacidad de una carretera corresponde a un concepto bastante bien definido, mientras que la capacidad de una población entera no posee una propiedad operacional clara aunque se pueda utilizar a nivel descriptivo. El hecho de que las relaciones simples agregadas pueden ponerse en evidencia deja abierta la cuestión relativa a su carácter operacional sin disminuir, por lo tanto, la ventaja de su carácter descriptivo.

\subsection{Procesos ¿determinísticos o estocásticos?}

Ilustraremos en la parte inferior el impacto de las fluctuaciones en los sistemas dinámicos deterministas. ${ }^{3}$

Consideremos un sistema compuesto de $N$ individuos, $x$ en el estado $X$ e $y$ en el estado $Y$ donde $N=x+y$. Supongamos que un individuo puede pasar de un estado al otro, ya sea de manera espontánea o de manera inducida. La transición espontánea corresponde, en marketing, a un proceso de comunicación masa $\rightarrow$ medio de comunicación, según el cual, un individuo en contacto con una fuerza externa tiene cierta probabilidad de cambiar de estado (por ejemplo, de pasar del estado no informado al estado informado). De manera simbólica los procesos de transición pueden representarse de la manera siguiente:

\footnotetext{
${ }^{3}$ El ejemplo de los sistemas taxonómicos de los botanistas o de los naturistas nos induce a pensar que los sistemas son en parte construidos y no desprovistos de prejuicios teóricos. La posible arbitrariedad en la selección de las características medidas restringe las clasificaciones que resultan. Otros sistemas implican, ante todo, un comportamiento completamente determinado por un conjunto bien definido de interacciones (tales como los sistemas dinámicos o termodinámicos); sin embargo, no pueden presuponer, de entrada, un comportamiento de conjunto.
} 


$$
\left\{\begin{array}{l}
X \stackrel{a}{\longrightarrow} Y \\
Y \stackrel{a^{\prime}}{\longrightarrow} X
\end{array}\right.
$$

Por otra parte, una transición puede hacerse también de manera inducida. Este proceso corresponde, en marketing, a un proceso de difusión de información de boca en boca. Así tenemos:

$$
\left\{\begin{array}{l}
X+Y \stackrel{b}{\longrightarrow} 2 X \\
X+Y \stackrel{b^{\prime}}{\longrightarrow} 2 Y
\end{array}\right.
$$

Las leyes elementales de la cinética nos enseñan que:

$$
\frac{d x}{d y}=a x-a^{\prime} y+b x y-b^{\prime} x y
$$

Supongamos que el problema sea simétrico: $a=a^{\prime}$ y $b=b$. En ese caso se obtiene la ecuación de evolución siguiente:

$$
\frac{d x}{d y}=a\left(\frac{N}{2}-x\right)
$$

Cuyo estado estacionario único y estable, st, es dado por $x^{s t}=y^{s t}=N / 2$. Podemos considerar también la versión estocástica de este modelo, considerando la ecuación de evolución de $\mathrm{P}(x, t)$, es decir, de la probabilidad que en el instante $t$ el número de individuos en el estado $X$ sea igual a $X$. En este caso, el número de individuos en cada uno de los estados es una variable aleatoria cuya evolución es proporcionada por la solución de un proceso de nacimiento y muerte. La dinámica se rige por la probabilidad de que un individuo en el estado $X$ transite durante un intervalo de tiempo dado, al estado $Y$. La probabilidad de que un individuo, en el estado $X$ en el instante $t$ cuando hay $y_{t}$ individuos en el estado $Y$, transite hacia el estado $Y$ durante el intervalo de tiempo $(t, t+\Delta t)$ viene dado por:

$$
\mathrm{P}_{\mathrm{x}, \mathrm{y}}(\mathrm{x}, \mathrm{t}) \Delta t+o(\Delta t)=(a+b y) \Delta t+o \Delta t
$$

La solución estacionaria del proceso estocástico correspondiente puede ser calculada analíticamente y se representa en la figura II. 


\section{Figura II \\ Solución estacionaria del proceso estocástico}

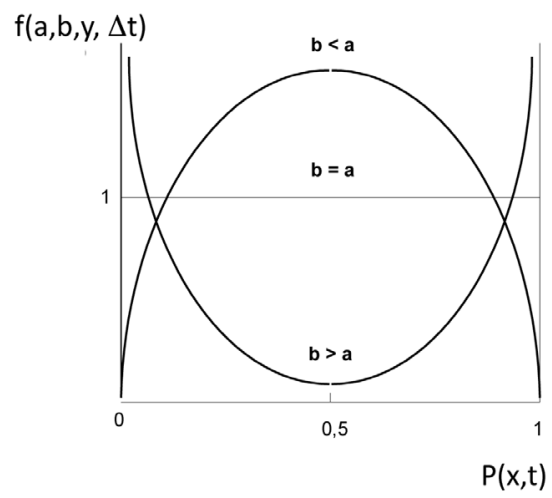

Fuente: Elaboración propia.

Si $b<a$, es decir, si el proceso de interacción no lineal es más reducido que el proceso de transición espontánea, la distribución estacionaria es máxima en $x^{\text {max }}=N / 2$, esto es, corresponde a la solución estacionaria del proceso determinista descrito por la ecuación (1). Por otra parte, si $b>a$, la distribución estacionaria privilegia los estados $x^{s t}=0$ y $x^{s t}=N$, esto significa que con $50 \%$ de probabilidad, la mayor parte de los individuos eligen uno de los estados $X$ o $Y$. Este resultado es nuevo vis-a-vis del obtenido a partir del modelo determinista (cf. ecuación 1). Por consiguiente, una vez que $b>a$, el caso mixto, (es decir $x^{s t}=x^{s t}=N / 2$ ) que es estable en el modelo determinista, es inestable en el modelo estocástico, en el sentido que una fluctuación cualquiera, que pudiera superar el tipo $X$, por ejemplo, corre el riesgo de amplificarse y de conducir el sistema a una situación tal que la mayoría de los individuos eligen, sea el estado $X$, sea el estado $Y$.

Es evidente que conocer si las fluctuaciones inherentes a todo sistema se van a amplificar o no es totalmente crucial. Además, el estudio de estabilidad del modelo determinista nos conduce a una intuición falsa en el caso presentado anteriormente, puesto que siempre sugiere la estabilidad del sistema mixto $x^{s t}=x^{s t}=N / 2$. Uno de los puntos claves que permiten la comprensión de la violación de la ley de los grandes números se aporta al examinar la forma funcional de las probabilidades de transición. De hecho, la forma de la ecuación (2) la presencia de interacciones no locales puesto que es el número total (indicado por $y$ ) quien interviene en la probabilidad de transición y no la densidad local. Podemos mostrar, como la intuición lo sugiere, que si en lugar de considerar la forma (2), se considera la forma siguiente (cuyas probabilidades de transición de- 
penden de las densidades locales) el sistema estocástico posee un estado estacionario que corresponde a la solución determinista:

$$
R_{x y}(x, t) \Delta t+o(\Delta t)=\left(a+b \frac{y}{N}\right) \Delta t+o(\Delta t)
$$

Es decir, se muestra que si la condición inicial es pura (por ejemplo, una fracción dada de individuos están en el estado $X$ con una probabilidad cercana a (1) entonces, la solución estacionaria del proceso estocástico correspondiente se aproxima al proceso determinista si el sistema es suficientemente grande en el sentido

$$
\lim _{N \rightarrow \infty}\left(\frac{x^{s}}{N}\right)=\frac{1}{2}
$$

Desgraciadamente, solamente en química es posible trabajar con densidades (tales que $x / N$ ) mientras que en ciencias sociales, sólo las interacciones a largo alcance son posibles. De hecho se habla cada vez más de globalización, de internet, de la epidemia planetaria de fusión de empresas, de manera tal que con frecuencia las distancias son eliminadas y el concepto de localidad tiende a desaparecer parcialmente. Esto implica en particular, que el estudio de un sub-sistema presenta problemas, puesto que las fronteras tienden a desaparecer, a volverse menos materiales y no localizadas en el espacio. Además, los resultados mostrados anteriormente sugieren que una consecuencia material de la globalización es que las fluctuaciones parásitas que han emergido espontáneamente en los sistemas sociales corren el riesgo de ser difícilmente destruidas por una ley de grandes números. Se puede entonces visualizar una doble implicación: el aumento de la globalización permite a unidades reducidas imponerse más fácilmente, beneficiándose de un mercado natural más importante.

Por otra parte, en un mundo global, el ojo de los otros es mucho más omnipresente y la posibilidad de desarrollar innovaciones de manera aislada es menos probable. Ahora bien, muchas de las innovaciones no son viables desde sus orígenes y necesitan, por lo tanto, protegerse temporalmente del mundo exterior para desarrollarse, así como las industrias nacientes necesitan barreras de protección al menos en el momento de su desarrollo inicial. Esta reflexión sobre la centralidad/globalidad es todavía incipiente pero, esperemos, llena de promesas, véase también el esquema estructuralista de la comunicación propuesta por Moles (1986). 


\section{El enfoque dinámico}

En esta sección se aportan tres elementos que ponen en evidencia la necesidad de incluir el enfoque dinámico en el análisis de los sistemas de transporte; el primero, se refiere a las limitaciones del enfoque estático para representar esquemas de organización básicos pero funcionales; el segundo aborda la posibilidad de incluir con este enfoque las consecuencias intertemporales en el análisis de fenómenos donde el antecedente, que lleva al sistema a un cierto estado, desempeña un papel determinante. Finalmente, el tercer elemento está vinculado con la posibilidad de incluir la aleatoriedad en las decisiones de las personas como una forma para representar la dinámica de un sistema de transporte.

\section{1. ¿Por qué el enfoque dinámico?}

Para introducir algunas limitaciones del enfoque estático se utiliza la siguiente anécdota: hace algunos años André de Palma fue invitado por Abraham Moles al departamento de sicología social de la Universidad de Estrasburgo; como joven físico esperaba dar un curso de matemáticas elementales para estudiantes de doctorado que deseaban formalizar algunas ideas novedosas desarrolladas por los fundadores de micro-psicología: Abraham Moles y Elisabeth Rohmer (1977). En lugar de este curso, Moles le pide exponer los trabajos más periféricos de la Escuela de Bruselas, presidida por el Nobel de Química (1976) Ilya Prigogine (1996), a la que estaba asociado en el marco de su tesis en física. En este equipo pluridisciplinario, Jean-Louis Deneubourg, de la Universidad Libre de Bruselas, había intentado (y logrado) aplicar la teoría de las estructuras disipativas para explicar los mecanismos de construcción de nidos de hormigas y de termitas (May, 1973).

De tal manera que las concepciones clásicas más precisas de los ingenieros de la construcción no son suficientes para describir la arquitectura de un hormiguero y responder a los siguientes cuestionamientos: ¿Dónde se encuentran los planos de los hormigueros y el de los nidos de las termitas?, ¿quién dirige a los obreros?, ¿cómo opera la división del trabajo?, ¿cómo operan los numerosos problemas de logística y los de secuencia de actividades?, como éstas, un sin número de preguntas en nuestra sociedad son bastantes complicadas, la razón de esta complejidad es que su formulación es muy ambiciosa. Ninguna hormiga podría comprender nuestras preguntas, así como ningún automovilista, que dispone de un plano urbano y del conjunto de desplazamiento de los otros usuarios de la red vial, será capaz, aún con la ayuda de las computadoras más potentes, de calcular soluciones comparables a las que se observan en realidad. De la misma manera, un individuo que llega a una ciudad que desconoce, 
tendrá que tomar un cierto número de decisiones para resolver sus necesidades. Aun teniendo acceso a la información sobre la localización de los servicios, los precios y la calidad de los bienes y servicios, le será difícil decidir sobre el conjunto de bienes y servicios de su cesto de consumo.

Estos ejemplos corresponden a una serie de problemas que cuando se plantean desde una perspectiva estática son difíciles a resolver. De hecho, son extraordinariamente arduos por la forma en que ellos son presentados, ya que no permiten al individuo utilizar los mecanismos elementales de retroalimentación. ${ }^{4}$

El ejemplo más clásico de retroalimentación es el del termostato que ajusta el flujo de calor en función de la temperatura. La causalidad clásica corresponde a la relación entre una causa (flujo de calor) y un efecto (temperatura ambiente). La retroalimentación se interesa en la relación inversa. El caso del termostato corresponde a una retroalimentación, dado que la variable de salida: la temperatura ambiente, actúa sobre la variable de entrada: el flujo de calor. Entendemos entonces porqué la regulación de la temperatura sin retroalimentación constituye un proceso extremadamente complejo. De tal manera que se trata de calcular el fenómeno de transmisión y de convección de calor, así como los fenómenos de pérdidas caloríficas que permitirán determinar ex-ante los flujos de calor necesarios para poder alcanzar un objetivo de temperatura ambiente determinado. Un ejemplo similar correspondería a la comparación entre un piloto automático que determina una trayectoria definida a partir de su lugar de destino y de su objetivo, y un autómata que ajustaría constantemente su trayectoria en función de las condiciones de circulación. Evidentemente, el segundo operador, que corresponde al comportamiento del automovilista, es mucho más fácil de modelar. En el caso de que el objetivo sea móvil, el primer tipo de estrategia necesita una anticipación del desplazamiento del mismo, en caso de conflicto o incidente, esto sería prácticamente imposible.

En resumen, esta relación de retroalimentación corresponde a un proceso de ensayo y de error, que efectivamente es más costoso en términos de gasto energético (en el caso del termostato), pero es también mucho más simple desde un punto de vista de cálculo y de memoria.

\subsection{Profecías auto-realizables y estados múltiples}

La construcción de nuevas vialidades tiene por objetivo el mejoramiento de las condiciones de circulación. Esta tautología es tan falsa en la práctica, como verdadera cuando se aborda superficialmente. La ley de Merton, que es conocida como Self-fullfilling prophecy (profecía autorrealiza-

\footnotetext{
${ }^{4}$ Este tipo de mecanismos fueron formalizados en un principio por los ingenieros/cibernéticos, como Wiener Von Neuman y Bertalanfy, para posteriormente ser retomados en las ciencias sociales.
} 
ble) proporciona argumentos lógicos contra la posibilidad de desarrollar predicciones. Si la oferta tiende a dar cuenta de la demanda, la demanda es entonces aumentada por la oferta. En efecto, el camino que satisface inicialmente una necesidad de comunicación generará rápidamente nuevas necesidades de la misma naturaleza. El especialista de transportes llama a este fenómeno, demanda inducida.

Estas relaciones, consideradas poco claras cuando fueron descubiertas, no lo son tanto si nos basamos en una simple lectura del diagrama de base de la economía que nos permite determinar los precios en un mercado en competencia (en nuestro caso el costo generalizado de desplazamiento al equilibrio) como la intersección entre la demanda (la disposición de los usuarios a pagar) y la oferta (la relación entre la cantidad de usuarios y la calidad del servicio ofrecido que se traduce en un costo generalizado para el individuo). En esta relación oferta-demanda, se puede mostrar que el aumento de la capacidad de las infraestructuras de transporte (nuevas vías o ampliaciones de ella) lleva a reducir el costo generalizado del usuario aumentando con ello la demanda de viajes (demanda inducida), lo que implica, que la infraestructura tendrá nuevamente problemas de saturación (Sánchez, 2004).

El fenómeno de la demanda inducida es más complejo cuando los usuarios o los compradores de un bien o servicio lo vuelven, por razones objetivas o subjetivas, más atractivo (anticongestión). Los especialistas en finanzas llaman a este fenómeno burbuja especulativa, que corresponde al aumento del precio de una acción inducida por el simple hecho que su adquisición, implica una inferencia del alza futura. Esta acción lleva por sí misma a decisiones de compra que constituyen efectivamente el aumento de precio. Una consecuencia de este fenómeno de profecía autorrealizable es la existencia de estados múltiples. De nuevo, la clave para la explicación de estos fenómenos es la descripción de los mismos con un modelo dinámico. Sólo un análisis de la dinámica que tome en cuenta las elecciones de nuestros antepasados permitiría entender la solución tomada por el sistema social presente. Las profecías autorrealizables y la existencia de estados múltiples en correlación, constituyen un argumento adicional en favor de la utilización del enfoque dinámico.

\subsection{Sistemas dinámicos y aleatoriedad}

\subsubsection{Motivación}

Se ha argumentado que los sistemas sin retroalimentación son de trato delicado, puesto que necesitan la resolución de problemas de tipo punto fijo, los cuales son difíciles de resolver. La retroalimentación permite 
desarrollar procedimientos de ensayo y error que conducen hacia métodos donde el error es permitido. Estos sistemas no están optimizados pero son adecuados desde el punto de vista del comportamiento.

Retomando el trabajo de los nidos de las hormigas, se puede decir que ciertamente las hormigas no optimizan su trabajo: un grupo de ellas pasan su tiempo, por descuido o inadvertencia, destruyendo el trabajo de sus colegas. Sin embargo, ningún sistema podrá resolver el trabajo de las hormigas utilizando tan poca potencia de cálculo y capacidad de memoria. En este sentido, las hormigas tienen mucho que enseñarnos, sobre todo a los ingenieros (Resnick, 2001).

\subsubsection{El modelo de elección dinámico}

Para ilustrar nuestro propósito, consideremos una red simple compuesta de un origen y un destino conectados por dos carreteras paralelas. Sea $x$ el número de personas utilizando la ruta $X$, e $y$ el de los usuarios que utilizan la ruta $Y$, donde $x+y=N$. El estado de equilibrio se obtiene una vez que el tiempo de recorrido sobre la ruta $X$, notado $t t_{X}(x)$ es idéntica al tiempo de recorrido sobre la ruta $Y, t t_{Y}(y)$. Si una solución interior existe, ésta satisface la relación $t t_{X}(x)=t t_{Y}(y)$. Será entonces bastante difícil para los usuarios elegir la mejor ruta sin posibilidad de coordinación. Por el contrario, si todos los individuos han elegido su ruta, excepto uno, este último habrá rápidamente efectuado su elección y ésta será la adecuada.

Consideremos ahora un caso intermedio en el cual una fracción de individuos ha efectuado su elección. Para aclarar la idea, se dice que estos individuos conservan para el día $w$ la elección efectuada el día $w$-1. Los otros individuos, que constituyen una fracción $R$ del total, deben efectuar su elección (esta puede ser la misma que el día precedente). Los $R^{*} N$ individuos pueden suponer que todos los otros individuos guardarán la elección realizada el día precedente. Este supuesto no es tan falso siempre y cuando el valor de $R$ no sea muy elevado. Sin embargo, comprendemos que este sistema posee algunas fuentes de inestabilidad dado que $\operatorname{los} R^{*} N$ individuos efectuarán la misma elección el día $w$, lo que podría conducir a comportamientos oscilatorios, caóticos y hasta explosivos en el caso de que $R$ sea demasiado elevado.

Resulta entonces que la estabilidad implica que el valor de $R$ debe ser extremadamente pequeño, en ese caso, la dinámica del sistema será demasiado lenta. Es posible salir de este callejón sin salida volviendo el modelo más realista y reconociendo explícitamente el hecho de que las preferencias de los usuarios son heterogéneas: estos no solamente poseen capacidad de cálculo y de predicción de variables, sino también responden a una serie de características no observables, diferentes a las del tiempo 
de recorrido. Lo anterior nos conduce a un modelo de elección de rutas probabilístico. En ese caso, notaremos $P_{X}\left(x_{w}, \mu\right)$ la probabilidad que un individuo, que ha decidido revisar su elección el día $w$, decide tomar la ruta $X$. El parámetro $\mu$ permite medir la heterogeneidad de las preferencias individuales. La probabilidad $P_{X}\left(x_{w}{ }^{*} \mu\right)$ es una función decreciente de $t t_{X}\left(x_{w}\right)$ y creciente de $t t_{Y}\left(y_{w}\right) \operatorname{con} x_{w}+y_{w}=N$.

La dinámica de día en día se escribe ahora:

$$
x_{w}=(1-R) x_{w-1}+R\left[y_{w-1} P_{X}\left(x_{w} \mu\right)-x_{w-1} P_{Y}\left(y_{w} \mu\right)\right]
$$

El estado estacionario de este sistema es dado por:

$$
x^{s t} P_{Y}\left(y^{s t}, \mu\right)=y^{s t} P_{X}\left(x^{s t}, \mu\right)
$$

Si los individuos son casi homogéneos ( $\mu$ pequeño), la solución estacionaria tiende hacia la solución clásica: $t t_{X}(x)=t t_{Y}(y)$. Por el contrario, cuando los individuos son bastante heterogéneos, la solución estacionaria tiende hacia la solución combinada $x^{s t}=y^{t t}=N / 2$. El estudio de estabilidad de la ecuación (4) muestra que, bajo condiciones de regularidad y de monotonía habituales, este sistema dinámico es mucho más estable:

El valor de R es pequeño. Este resultado es interesante, dado que muestra el proceso heurístico de cálculo de un estado estacionario (4), no converge, si el número de individuos susceptibles de modificar su elección cada día es suficientemente pequeño. De hecho, los individuos suponen que el día $w$ la elección de los otros individuos son idénticos a aquéllos efectuados (y observados) el día $w$-1. Esta aproximación es mucho más correcta cuando el valor de $R$ es pequeño.

El valor de $\mu$ es grande. El caso homogéneo se obtiene tomando el valor más pequeño del parámetro $\mu$. En este caso, todos los individuos en posición de elección optarán, con una probabilidad de 100\%, por la ruta cuyo tiempo de trayecto haya sido el más reducido el día anterior. Por el contrario, cuando el valor de este parámetro es grande, tenemos que otros factores diferentes al tiempo de recorrido entran en el proceso de elección del individuo. Por ejemplo, la elección de la ruta podría estar afectada por el número de semáforos, por los espacios verdes a recorrer, por la calidad de la carretera, etc. Esta heterogeneidad, implica que los individuos están menos sujetos a su propio proceso de elección, y por lo tanto, refuerzan la estabilidad del sistema. Se ha mostrado, en diferentes contextos, la pertinencia de la heterogeneidad sobre la existencia de un estado de equilibrio. Este efecto esta plasmado, de hecho, en el marco del cálculo de un equilibrio de Nash estático (Anderson et al., 1992 y De Palma et al., 1985). 
¿Cuáles son las enseñanzas que podemos extraer de éste análisis? De entrada, debemos notar que los dos efectos, explicados muy simplemente en el caso de dos carreteras en paralelo, se reencuentran desde un punto de vista cualitativo para el caso de simulaciones de grandes redes de transportes compuestos de varios miles de arcos. La enseñanza, en este sentido, es por lo tanto robusta. Este análisis muestra también que las diferentes piezas de un modelo deben ser combinadas con un grado de precisión comparable. En efecto, un modelo de elección exageradamente preciso ( $\mu$ demasiado pequeńo), no aporta valor agregado, además puede poner en riesgo la estabilidad del sistema cuando las otras partes del modelo son de baja calidad. Es decir, cuando el valor de $R$ es bastante reducido, lo que implica una calidad baja del proceso de predicción del estado del tráfico para el día $w$, dado que éste se basa en el estado del tráfico del día $w-1$ ).

En el folclor del científico, podemos entender el hecho de que un modelo, por muy bueno que sea, no podrá aportar resultados correctos si los datos utilizados son erróneos. El resultado enunciado anteriormente será algo diferente, lo que lleva a la afirmación de que las diferentes piezas de un modelo deben poseer el mismo grado de rigor, y que el sistema puede ser inestable si una de las piezas posee un grado de precisión mucho más importante que las otras. El segundo resultado es que la descripción dinámica de un sistema permite hacer uso de procesos cognitivos infinitamente más simples que el caso de los modelos estáticos. De alguna manera se puede decir que el tiempo permite desenredar el problema propuesto, siguiendo un hilo conductor que induce naturalmente un orden en el desarrollo de las tareas elementales. El carácter ordenado del tiempo posee quizás una riqueza natural que permanece todavía por explorar. ${ }^{5}$

\section{Conclusiones}

Las implicaciones en el ámbito de la investigación y la práctica en materia de modelación de sistemas de transporte han motivado a realizar un análisis crítico y conceptual sobre las limitaciones de las metodologías y enfoques utilizados actualmente. Dicho análisis se articuló a través de cuatro ejes: la modelación del comportamiento del individuo según la perspectiva de sicología social y en micro-sicología, el enfoque clásico de análisis del comportamiento utilizado en economía, las limitaciones de la formulación determinista con respecto a la estocástica, y finalmente, los

\footnotetext{
${ }^{5}$ Evidentemente, aquí sólo hemos considerado el tiempo irreversible, el tiempo entrópico y fecundo que posee una memoria, y no el tiempo hermético, irreversible y estático de la mecánica racional.
} 
contrastes entre el enfoque de modelación estático y dinámico. De este análisis se indicaron algunas líneas emergentes de investigación cuyo desarrollo permitiría subsanar algunas de las limitaciones referidas.

Por otra parte, dicho análisis permite obtener argumentos que llevan a concluir que la modelación de los sistemas de transporte debería contar con las siguientes características: 1) Desagregado, es decir, que caracterice comportamientos individuales más que comportamientos medios; 2) Estocástico, que reconozca explícitamente el hecho de que los individuos son diferentes y que existe una gran cantidad de factores y variables que no son observables desde el punto de vista del modelador; 3) Dinámico, que se base en leyes heurísticas que permitan a los individuos la exploración de estrategias por un proceso iterativo de ensayo y error.

Así también se ha enunciado la idea según la cual, no solamente las descripciones agregadas son peligrosas, sino que los modelos desagregados podrían ser incorrectos en el largo plazo, dado que las preferencias cambian de manera exógena y desconocida para el modelador. Una vía de investigación prometedora consiste en buscar las variantes que son susceptibles de explicar estas preferencias, vía que es poco explorada en el área de los transportes.

\section{Bibliografía}

Anderson, Simon, André de Palma y Jean Francois Thisse (1992), Discrete choice theory of product differentiation, MIT Press, Cambridge.

Ben-Akiva, Moshe y Stephen Lerman (1985), Discrete choice analysis: theory and applications to travel demand, MIT Press, Masachussets.

Beckmann, Martin Josef, McGuire, Chris y Winsten Christoper B. (1956), Studies in the economics of transportation, Yale University Press, New Haven.

Boursin, Jean Louis (1995), Des préférences individuelles aux choix collectives, Economica, Paris.

Bowman, John y Ben-Akiva, Moshe (2001), "Activity-based disaggregate travel demand model system with activity schedules", Transportation Research Part A: Policy and Practice, 35 (1), Pergamon, Amsterdam, pp. 1-28. 
Braess, Dietrich, Anna Nagurney y Tina Wakolbinger (2005), "On a paradox of traffic planning", Transportation science, 39 (4), Informs, Hanover, pp. 446-450.

Braess, Dietrich (1968), "Über ein paradoxon aus der verkehrsplanung”, Unternehmensforschung 12, Würzburg, Heidelberg, pp. 258-268.

Carrasco, Juan-Antonio y Eric Miller (2009), "The social dimension in action: A multilevel, personal networks model of social activity frequency between individuals", Transportation Research Part A: Policy and Practice, 43-1, Pergamon, Amsterdam, pp. 90-104.

Daganzo, Carlos (1994), "The cell transmission model: A dynamic representation of highway traffic consistent with the hydrodynamic theory", Transportation Research Part B: Methodological, 28-4, Pergamon, Amsterdam, pp. 269-287.

De Palma, André, Victor Ginsbugh, Papageorgiou Yorkos y Jean Francois Thisse (1985), "The principle of minimum differentiation holds with sufficient hetorogeneity", Econometrica, 53, Wiley, Nueva York, pp. 767-781.

De Palma, André y Serge Pahaut (1989), "Phenomènes et symboles", en La physique des sciences de l'homme, Ediciones oberlin, Strasbourg, pp. 105-108.

De Palma, André (1998), "Individual and Collective Decision Making: Application to Urban Transportation”, en Tommy Garling, Thomas Laitila y Kerstin Westin (eds.), Theorical Foundations of Travel Choice Modeling, Pergamon Press, Nueva York, pp. 33-50.

De Palma, André y Serge Pehaut (1999), "Les modèles de decision et la politique comme processus collectif: Applications aux sistèmes de transport urbain", documento de trabajo. THEMA-Universidad de Cergy-Pontoise, Cergy, 16 pp.

De Palma, André y Fabrice Marchal (2002), "Real cases applications of fully dynamic metropolis Tool-Box: An advocacy for largescale mesoscopic transportation systems", Network and spatial economics, 2, Springer-Verlag, Berlín, pp. 347-369. 
Etemma, Dick y Harry Timmermans (1997), Activity-based approaches to travel analysis, Pergamon, Oxford.

Gazis, Denos (2002), Traffic theory, Springer-Verlag, Berlín.

Hall, Randolph (2003), “Transportation science”, en Randolph Hall (ed.), Handbook of transportation science, international series in operations research \& management science, 56, Springer-Verlag, Alemania, pp. 1-5.

Hensher, David y William Grenne (2010), Modelling ordered choices, a primer, Cambridge, Reino Unido.

Lecourt, Dominique (2010), La filosofía de las ciencias, trad. Óscar Sánchez, Universidad Autónoma del Estado de México, Toluca.

May, Robert (1973), Stability and Complexity in Model Ecosystems, Princeton University Press, Princeton.

Mohring, Hebert (1999), "Congestion”, en Essays in Transportation Economics and Policy: A Handbook in Honor of John R. Meyer, The Brooking Institution, Washington DC., pp. 181-222.

Moles, Abraham (1986), Théorie Structurale de la Comunication et societé, Mason, Paris.

Moles, Abraham y Elisabeth Rohmer (1977), Théorie des actes: vers une écologie des actions, Casternam, Paris.

Moreno, Jose Luis (1970), Fondements de la sociométrie, Presses Universitaires de France, Paris.

Ortúzar, Juan de Dios y Luis Willumsen (2001), Modelling Transport, Wiley, New York.

Ortúzar, Juan de Dios y Óscar Sánchez (2004), Métodos y modelos en la planeación del transporte, Universidad Autónoma del Estado de México, Toluca.

Prigogine, Ilya (1996), La fin des certitudes: temps, chaos et les lois de la nature, Editions Odile Jacob, Paris. 
Resnick, Mitchel (2001), Tortugas, termitas y atascos de tráfico, exploraciones sobre micromundos masivamente paralelos, Editorial Gedisa, Barcelona.

Samuelson, Paul Anthony (1952), "Spatial price equilibrium and linear programming", The American Economic Review, 42-3, American Economic Association, Pittsburgh, pp. 283-303.

Sánchez, Óscar (2004), "Impacto vial inducido por la construcción de la terminal norte de la ciudad de Toluca", Cuadernos de Investigación 33, Universidad Autónoma del Estado de México, Toluca.

Schelling, Thomas (1980), La tyrannie des petites décisions, Presses Universitaires de France, Paris.

Stopher, Peter y Cherril Stecher (2006), Travel survey methods: quality and future directions, Elsevier, Amsterdam.

Vickrey, William (1969), "Congestion theory and transportation investments", American Economic Review, papers and proceedings, 59, American Economic Association, Pittsburgh, pp. 251-261.

Wachowicz, Monica (2010), Movement-aware applications for sustainable mobility: Technologies and approaches, Information science reference, New York.

Wasserman, Stanley y Katherine Faust (1999), Social network analysis: methods and applications, Cambrige University Press, Cambridge.

Recibido: 13 de julio de 2009. Reenviado: 18 de octubre de 2011. Reenviado: 6 de febrero de 2012. Aceptado:18 de marzo de 2012.

Óscar Sánchez-Flores es doctor en ciencias económicas (Université de Cergy-Pontoise, Francia). Actualmente es profesor investigador en el departamento de posgrado de la Facultad de Ingeniería de la Benemérita Universidad Autónoma de Puebla. Su línea de investigación actual es la modelación de sistemas de transporte. Ha publicado recientemente: en coautoría, "Factores que integran la noción de calidad de servicio en el transporte público de pasajeros: el caso de un corredor urbano en la ciu- 
dad de Toluca”, Economía, Sociedady Territorio, X (32), El Colegio Mexiquense, A.C., Zinacantepec, pp. 49-80 (2010); en coautoría, "Are static and dynamic planning approaches complementary? A case study for travel demand management measures", en Bohme et al. (eds.), Lectures notes in computer science 3473, Springer-Verlag, Berlín, pp. 252-264 (2006); en coautoría, Automatic bottleneck detection based on macroscopic traffic data: An application to Paris highway network, en Bohme et al. (eds.), Lectures notes in computer science 3473, Springer-Verlag, Berlín, pp. 236251 (2006). Ha traducido del francés el libro La filosofía de las ciencias de P. Lecourt, Publicaciones de la Universidad Autónoma del Estado de México (2010).

André de Palma es doctor en física por la Universidad Libre de Bruselas, Bélgica y doctor en economía por la Universidad de Burgońa, Francia. Actualmente es profesor del departamento de Economía de la L'Ecole Polytechnique y profesor del departamento de economía y gestión de la Ecole Normale Superieure de Cachan, Francia. Sus líneas de investigación se inscriben en diversas áreas como finanzas, economía del transporte, teoría del riesgo. Ha publicado recientemente: en coautoría, "Traffic congestion pricing methodologies and technologies", Transportation Research Part C: Emerging Technologies, 19-6, Pergamon, Amsterdam, pp. 1377-1399 (2011); en coautoría, "Assessing transport investmentsTowards a multi-purpose tool”, Transportation Research Part B: Methodological, 44-7, Pergamon, Amsterdam, pp. 834-849 (2010); en coautoría con, "The merits of separating cars and trucos", Journal of Urban Economics,64-2, Elsevier, Oxford, pp. 340-361 (2008); en coautoría, "Standardized versus customized portfolio: a compensating variation approach", Annals of operation research, 165-1, Springer-Verlag, Berlín, pp. 161-185 (2009); en coautoría, "Risk aversion in expected intertemporal discounted utilities bandit problems. Theory and decision, 67-4, Springer-Verlag, Berlín, pp. 433-440 (2009). 